\title{
Development and preliminary validation of a new screening questionnaire for identifying atopic children
}

This article was published in the following Dove Press journal:

Pediatric Health, Medicine and Therapeutics

21 September 2017

Number of times this article has been viewed

\author{
Marta Sacchetti ${ }^{1,2}$ \\ Ilaria Baiardini ${ }^{3}$ \\ Loredana Chini ${ }^{4}$ \\ Viviana Moschese ${ }^{4}$ \\ Alice Bruscolini ${ }^{2}$ \\ Alessandro Lambiase ${ }^{2}$ \\ 'Cornea and Ocular Surface Unit, \\ IRCCS-Ospedale San Raffaele di \\ Milano, Milan, ${ }^{2}$ Department of Sense \\ Organs, Sapienza University, Rome, \\ ${ }^{3}$ Allergy and Respiratory Diseases \\ Clinic, DIMI, University of Genoa, \\ IRCCS AOU San Martino-IST, Genoa, \\ ${ }^{4}$ Pediatric Allergology and Immunology \\ Unit, Policlinico Tor Vergata, University \\ of Rome Tor Vergata, Rome, Italy
}

Background: Allergic diseases represent a frequent and increasing condition affecting children. A screening questionnaire allowing an easy identification of children with symptoms of allergic diseases may improve management and clinical outcome. The aim of this study was to develop and validate an easy-to-use screening questionnaire to detect children requiring further allergological evaluations.

Methods: A 10-item questionnaire, evaluating the presence and the history of the most frequent allergic conditions affecting children, including allergic asthma, allergic rhinitis and conjunctivitis, food allergy, and atopic dermatitis, was developed and administered to 214 parents of children from 5 to 10 years of age (163 with allergic disease and 51 healthy, nonallergic children). Validation was performed by Pearson's correlation between the clinical diagnosis and the responses to the questionnaire. Internal consistency was computed by Cronbach's alpha correlation coefficient. Sensitivity and specificity of the novel questionnaire were assessed by the receiver operating characteristic (ROC) curve.

Results: Validation analysis of the new children atopy (ChAt) questionnaire showed good internal consistency with a Cronbach's alpha of 0.757 . Responses to the items evaluating the presence of individual allergic conditions significantly correlated with the clinical diagnosis $(p<0.001)$. The ROC curve showed an area of 0.956 and identified a cutoff value $>2$ of the ChAt questionnaire total score for detection of allergy (sensitivity $=0.92$ and specificity $=0.902$ ).

Conclusion: The novel ChAt questionnaire represents a simple tool able to detect the presence of all major allergic diseases in a pediatric population allowing an early identification of allergic multimorbidity and potentially facilitating clinical management.

Keywords: allergy, questionnaire, children, screening, validation

\section{Introduction}

Allergic diseases represent a leading cause of chronic illness in children, with up to $30 \%$ of pediatric population affected by some form of atopic disease. ${ }^{1-3}$ Moreover, the prevalence of allergy seems to have dramatically increased over the past 25 years, particularly in Western industrialized countries. ${ }^{4,5}$ Among the Italian children, prevalence of allergic disorders varies from $20 \%$ for rhinitis to $10 \%$ for eczema/dermatitis, $9 \%$ for asthma, and $8 \%$ for food allergy. ${ }^{6-8}$

Since allergic inflammation involves different target tissues (conjunctiva, nose, lung, and skin) causing allergic conjunctivitis (AC), rhinitis (AR), asthma symptoms, and atopic eczema/dermatitis, evaluation of the prevalence of allergic diseases requires that any form of allergic response should be identified and addressed. ${ }^{9}$ In fact, atopic conditions are often comorbid: $20 \%-40 \%$ of patients with AR are reported to have
Correspondence: Alessandro Lambiase Department of Sense Organs, University Sapienza of Rome, Viale del Policlinico, 15500161 Rome, Italy

Tel +390649975357

Fax +3906 49975357

Email alessandro.lambiase@uniroma I.it 
asthma, and $30 \%-90 \%$ of patients with asthma have AR. ${ }^{10,11}$ In addition, it has been proposed that early diagnosis and treatment should improve clinical outcome of allergy in children by controlling the progression toward the most severe forms, such as allergic asthma. ${ }^{4}$ Therefore, screening instruments, allowing an early and simple identification of children with suspected allergic conditions, may improve patients' health and clinical outcomes. ${ }^{12,13}$ Questionnaires are useful screening tools with the advantages of being noninvasive, low cost, and easy to administer and provide quick results. ${ }^{13-15}$ Allergic screening questionnaires for school children have been developed for the detection of individual allergic conditions to improve early identification and management of allergic diseases. ${ }^{12,16-18-}$ Most of them are focused primarily on detection and/or prediction strategies of asthma, with comorbid allergic diseases, including rhinitis and conjunctivitis, used to increase the specificity of allergic diseases detection. ${ }^{17-22-}$ In this study, to identify the children with symptoms of all the major allergic disease types, we developed and validated a novel, simple, and easy-to-use children atopy (ChAt) questionnaire that addresses the primary allergic conditions.

\section{Methods}

Children between 5 and 10 years of age were recruited consecutively by the Pediatric Allergology and Immunology Unit, Policlinico Tor Vergata, University of Rome Tor Vergata, Italy, for the development and initial validation phases of the new screening questionnaire for the detection of allergic diseases in children (ChAt questionnaire).

The study was conducted in accordance with the principles embodied in the Declaration of Helsinki of 1965 (as revised in Brazil 2013). All parents of the participants provided written informed consent, and patient anonymity was preserved using methods approved by the Local Ethics Committee of the University of Rome Tor Vergata.

Diagnosis of allergic diseases including AC, AR, allergic asthma, atopic dermatitis, and food allergy was based on clinical history, objective examination, and allergological exams (including skin prick tests and specific Immunoglobulin (Ig) E detection).

\section{ChAt screening questionnaire development}

The development of ChAt screening questionnaire included the following steps. ${ }^{23,24}$

1. Item generation. The aim of this phase was to identify the items and conditions to be included in the screening questionnaire. A preliminary list of items was developed on the basis of the following sources:

- An international literature review was performed through a computerized search in the Medline database to identify existing screening questionnaires for allergic diseases in children by using the following keywords/search terms: atopy*, allergy*, asthma*, allergic conjunctivitis*, allergic rhinoconjunctivitis* atopic dermatitis*, food* and allergy, children questionnaire and screening questionnaire.

- A panel of four specialists (VM, LC, MS, AL) were required to indicate the most frequent allergic conditions affecting children and how to identify them through simple questions for administration to the parents.

2. Item reduction. A qualitative selection was performed from the preliminary list of items by eliminating those which were redundant, difficult to understand, or ambiguous. The resulting screening questionnaire included 10 items aimed at identifying a history of allergic diseases and the presence of specific symptoms of allergy: 1) previous diagnosis of allergic diseases, 2) previous use of antiallergic drugs, 3) presence of symptoms of AC, 4) presence of symptoms of $A R, 5)$ presence of symptoms of asthma, 6) presence of symptoms of atopic dermatitis, 7) history of severe allergic conditions such as anaphylaxis, 8) perennial and/or seasonal presentation of allergic symptoms, 9) family history of allergic diseases, and 10) history of food allergy including elimination diet (Table 1).

Cognitive debriefing interviews with 10 parents of allergic children were performed to verify that the items were clear and easy to understand. The screening questionnaire was administered to the patient population for initial validation.

\section{Validation of the new ChAt questionnaire}

Two-hundred and fourteen parents of children between 5 and 10 years of age completed the screening questionnaire. Onehundred and sixty-three children were diagnosed with one or more allergic diseases by allergological exam, and in 51 children allergic diseases were excluded. This group was utilized as the healthy control group for the validation of the ChAt questionnaire. The aim of the validation phase was to evaluate the following psychometric properties of the new instrument:

- Validity: using the Pearson correlation coefficient, we evaluated the relationship between the clinical diagnosis and the responses to the questionnaire. ${ }^{13}$ 
Table I ChAt questionnaire items and score values

\begin{tabular}{|c|c|c|}
\hline \multirow{2}{*}{$\begin{array}{l}\text { Screening questionnaire for allergic diseases in children } \\
\text { (to be completed by parents) }\end{array}$} & \multicolumn{2}{|c|}{ Response scores } \\
\hline & Yes & No \\
\hline $\begin{array}{l}\text { I. Was your child diagnosed with an allergic disease? } \\
\text { If yes, please specify: } \\
\text { - Allergic asthma } \\
\text { - Allergic rhinitis } \\
\text { - Allergic conjunctivitis } \\
\text { - Hives } \\
\text { - Atopic dermatitis } \\
\text { - Food allergy (with elimination diet) } \\
\text { - Anaphylaxis }\end{array}$ & I & 0 \\
\hline 2. Did your child ever use drugs for an allergic disease? (e.g., antihistamines, corticosteroids, etc.) & I & 0 \\
\hline 3. Does your child often show red eyes, tearing, and itching? & I & 0 \\
\hline 4. Does your child sneeze often or does he/she have nasal itching and discharge? & I & 0 \\
\hline $\begin{array}{l}\text { 5. Did your child ever complain of breathing problems? (e.g., shortness of breath, chest tightness, wheezy } \\
\text { breathing, coughing, or itchy throat) }\end{array}$ & I & 0 \\
\hline 6. Does your child often have dermatitis with itching? & 1 & 0 \\
\hline 7. Did your child ever suffer with severe allergic reactions or anaphylactic shock? & I & 0 \\
\hline $\begin{array}{l}\text { 8. Does your child have any of the above symptoms? } \\
\text { If yes, please specify if: } \\
\text { - Seasonal: spring/summer or autumn/winter } \\
\text { - Throughout the year }\end{array}$ & I & 0 \\
\hline 9. Does your child have relatives who suffer or have suffered of an allergic disease? & I & 0 \\
\hline $\begin{array}{l}\text { 10. Did your child ever have allergic reactions to food? } \\
\text { If yes, please specify: } \\
\text { - Asthma } \\
\text { - Dermatitis } \\
\text { - Rhinoconjunctivitis }\end{array}$ & & \\
\hline Currently, is he/she under an elimination diet? & I & 0 \\
\hline
\end{tabular}

Abbreviation: ChAt, children atopy.

- Internal consistency: a measure of the homogeneity of a scale was computed using the Cronbach's alpha correlation coefficient, and test-retest reliability, was evaluated by using intraclass correlation coefficient (ICC). Measurements with reliability of 0.70 or more have been recommended for the purpose of comparing groups. ${ }^{14}$

Discriminant validity was evaluated by comparing the scores of allergic patients. Sensitivity and specificity of the new ChAt questionnaire were assessed by the receiver operating characteristic (ROC) curve in the entire population included in this study. The type and number of allergic diseases affecting the children were related to the ChAt total score by Sperman's rho test.

The statistically significant cutoff value was set at $p<0.05$. The entire statistical analysis was conducted by using SPSS software version 18.0.

\section{Results}

Parents of one-hundred sixty-three allergic $(\mathrm{N}=163$, mean age $7.2 \pm 1.1$ years, 63 girls and 100 boys) and non allergic
( $N=51$, mean age $7 \pm 0.9$ years, 19 girls and 32 boys) children (mean age $7.2 \pm 1$ years, 132 boys and 82 girls) completed the screening questionnaire.

Among the allergic children, 97 (59.5\%) patients had a clinical diagnosis of AR, 51 (31\%) of AC, 57 (35\%) of asthma, 68 (42\%) of atopic dermatitis, and 11 (7\%) of food allergy. Eightynine of the $163(55 \%)$ allergic patients were diagnosed with more than one allergic condition ( 59 had been diagnosed with 2 different allergic diseases, 27 with 3 , and 3 with 4; Table 2).

The completion of the questionnaire by parents of allergic children included in the study is summarized in Figure 1.

Validation analyses were undertaken considering the group of allergic patients $(\mathrm{N}=163)$.

1. Validity. Item and scale scores were oriented so that $\mathrm{No}=$ 0 and Yes $=1$ (Table 1); the sum of scores ranges between 0 and 10 , and the higher the score, the higher probability to have an allergic disease.

2. Internal consistency. The screening questionnaire demonstrated satisfactory Cronbach's alpha value of 0.757 and ICC of 0.84 . 
Table 2 Clinical characteristics of allergic population included in the study

\begin{tabular}{ll}
\hline Clinical characteristics & Allergic children $(\mathbf{N}=163)$ \\
\hline Age (years) & $5-10$ \\
Range & $7 \pm 0.9$ \\
Mean \pm SD & \\
Gender & 100 \\
$\quad$ Males & 63 \\
Females & $74(45 \%)$ \\
Single allergic condition & $89(55 \%)$ \\
Multiple allergic conditions & \\
Allergic diseases diagnosis & $97(59.5 \%)$ \\
Allergic rhinitis & $51(31 \%)$ \\
Allergic conjunctivitis & $57(35 \%)$ \\
Asthma & $68(42 \%)$ \\
Atopic dermatitis & $11(7 \%)$ \\
Food allergy & \\
Skin prick tests & $16(10 \%)$ \\
Negative & $126(77 \%)$ \\
Positive & $21(13 \%)$ \\
Not done &
\end{tabular}

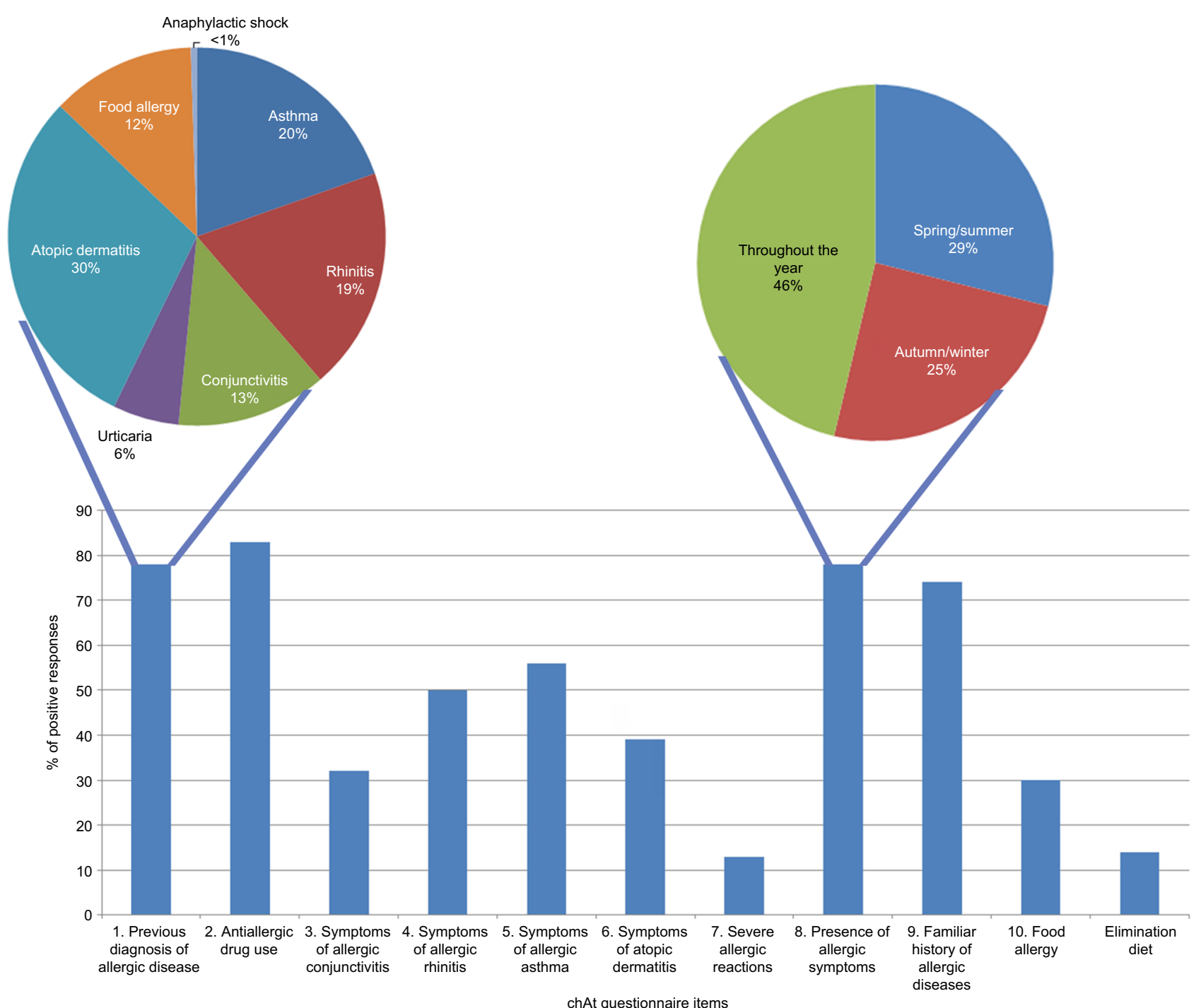

A strong correlation was observed between the clinical diagnosis and the responses to the questionnaire. Specifically, the following items showed strong correlation:

- Item 3 - clinical diagnosis of $\mathrm{AC}$ vs symptoms of $\mathrm{AC}$ $(p<0.001, R=0.330)$

- Item 4 - clinical diagnosis of AR vs symptoms of AR $(p<0.0001, R=0.467)$

- Item 5-clinical diagnosis of allergic asthma vs symptoms of allergic asthma $(p<0.0001, R=0.527)$

- Item 6 - clinical diagnosis of atopic dermatitis vs symptoms of atopic dermatitis ( $p<0.0001, R=0.483$ )

- Item 9 - familiar history of allergic disease collected by physician vs positive response to familiar history in the questionnaire $(p<0.0001, R=0.368)$

- Item 10 - presence of food allergy diagnosed by allergologist vs positive response to food allergy with elimination diet in the questionnaire $(p<0.0001, R=0.370)$

Figure I Percentage of positive responses to the ChAt questionnaire items. 
The ROC curve was calculated including the entire population of allergic and nonallergic children. Results showed an area of 0.956 and suggested that a cutoff score of $>2$ was able to accurately identify the allergic population with a sensitivity value of 0.920 and specificity of 0.902 (Figure 2).

Patients with a diagnosis of allergy showed an average ChAt questionnaire score of $5.2 \pm 2$, while nonallergic patients showed an average of $1.2 \pm 1.2$, confirming that the cutoff total score $>2$ is able to identify the allergic population. In addition, the number of allergic diseases affecting the children was significantly related to the ChAt total score $(p<0.001, R=0.695)$.

ChAt questionnaires with total score $>2$ identified a population with the possible presence of allergy which requires further clinical investigation for allergic disease, with a sensitivity of $92 \%$ (95\% CI: $86.75 \%$ to $95.69 \%)$ and a specificity of $90 \%$ (95\% CI: $78.59 \%$ to $96.74 \%$ ), a positive likelihood ratio (LR) of 9.39 (95\% CI: 4.08 to 21.61$)$ and negative LR of 0.09 (95\% CI: 0.05 to 0.15 ).

\section{Discussion}

Atopic diseases represent a substantial burden on the health care system, and great attention should be paid to identify children with allergic diseases in a timely manner and to provide prompt treatment and management of allergic diseases. ${ }^{25}$ Several screening tools, including screening questionnaires and prediction rules, have been developed to detect specific allergic conditions and the risk of progression toward more severe allergic conditions such as asthma. ${ }^{17,18,24}$ Since a simple and ready-to-use tool for the identification of children with

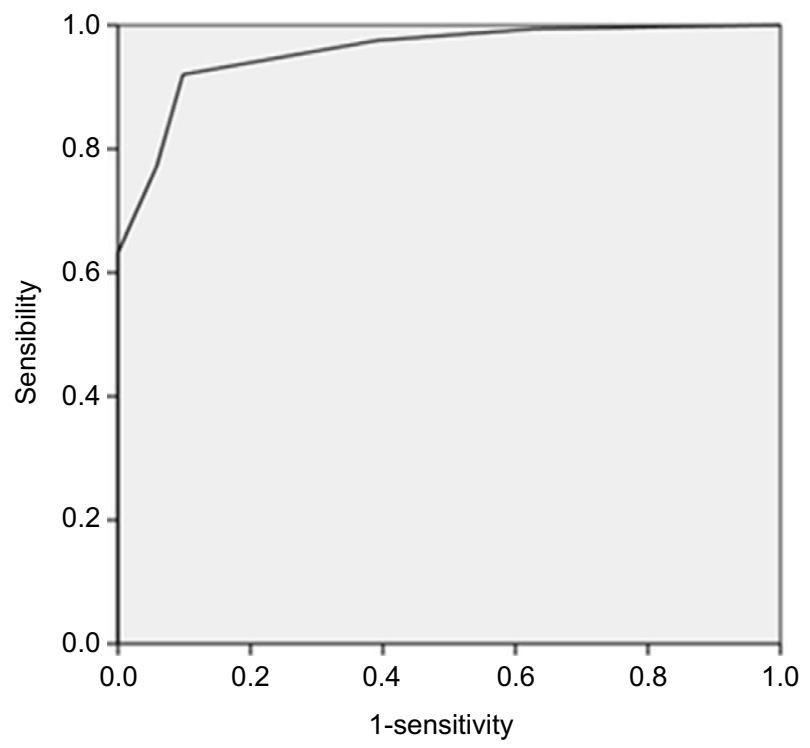

Figure 2 Receiver operating characteristic (ROC) curve of ChAt questionnaire scores for identification of the presence of allergic diseases.

Abbreviation: ChAt, children atopy. allergic symptoms including all the most common symptoms of allergic diseases is not currently available, in this study we developed and validated a new 10-item screening questionnaire to identify children with allergic symptoms requiring further clinical evaluation. This questionnaire represents a useful tool for the detection of children with suspicion of allergy in epidemiological, screening studies or in general practice.

Most studies commonly use questionnaires to collect epidemiological and clinical data. Moreover, self-administration of questionnaires avoids interviewer bias. ${ }^{17,18,26,27}$ On the other hand, the main issue in the validation process of selfadministered questionnaires is the capability to understand the items and to appropriately answer them. To facilitate this process, the ChAt questionnaire has been developed with well-defined, objective, and easily understandable questions for parent self-administration. In addition, respondents were selected and screened from the same geographical area (Rome, Italy). ${ }^{28}$

The ChAt questionnaire includes an item exploring the previous diagnosis of allergic conditions, with specification of the previous diagnosis, and an item about the previous use of antiallergic drugs to aid in the identification of allergic children. The other five items explore the presence of the most frequent symptoms associated with $\mathrm{AC}, \mathrm{AR}$, asthma, atopic dermatitis, and food allergy including elimination diet. The responses to these items showed a significant correlation with the clinical diagnosis of the specific allergic condition made by the allergologist, suggesting the ability of the items to identify the presence of the symptoms of specific disease. An additional three items evaluate the history of severe allergic reactions such as anaphylaxis, seasonal and/or perennial symptoms' presentation, and familial history of allergy, adding important information for the identification of allergic children.

The ChAt score was calculated by the sum of the "YES" responses to the 10 items resulting in a total score ranging from $0-10$, with the higher total score indicating a higher probability of allergy. The ROC curve analysis showed that a ChAt questionnaire total score $>2$ was able to identify children having allergy with high levels of sensitivity (92\%) and specificity $(90 \%)$.

Therefore, this study describes the development and preliminary validation of a simple, standardized, 10-item screening questionnaire that is able to identify atopic children who should be evaluated for the presence of allergic diseases and allergic comorbidities. The ChAt questionnaire shows the limitations characteristic of the use of questionnaires for disease screening, which includes the need to demonstrate the validity in different settings, in terms of 
sample population, social conditions, and geographic distribution, and to standardize the clinical diagnosis of the specific allergic conditions to be used as "gold standard." ${ }^{12-15,17-19,28}$ An epidemiological study is currently ongoing in a large population of schoolchildren to confirm the validity of the ChAt questionnaire at identifying children with allergic symptoms and allowing an early diagnosis of "unknown" cases.

Assessing allergic diseases at a community level using simple questionnaires, such as ChAt, may be a first step to change patient behaviors and to target educational initiatives aimed at improving the allergic disease outcomes. In fact, not only is poor disease control a negative prognostic factor for allergic disease progression, but it may also be associated with low continuity of care due to a poor doctor-patient relationship, negative attitudes, and concerns about the use of medication, and little understanding of the disease. ${ }^{10,29}$ The consequences of such a scenario are detrimental for atopic children, their families, and the health care system in general.

To conclude, the ChAt questionnaire represents a useful tool to identify children with suspicion of multiple atopic conditions and, in turn, to substantially contribute to further enhance the treatment and clinical management of allergic diseases. In addition, the ChAt questionnaire may facilitate the collection of data on school children population, which could be useful to save time and serve as useful resource in large epidemiological studies.

\section{Acknowledgment}

This study was supported by Italian Ministry of Health grant for Young Researchers (GR-2009-1595946).

\section{Disclosure}

The authors report no conflicts of interest in this work.

\section{References}

1. Downs SH, Marks GB, Sporik R, et al. Continued increase in the prevalence of asthma and atopy. Arch Dis Child. 2001; 84: 20-23.

2. Skoner DP. Allergic rhinitis: definition, epidemiology, pathophysiology, detection, and diagnosis. J Allergy Clin Immunol. 2001;108(1 Suppl): S2-S8.

3. Quercia O, Incorvaia C, Puccinelli P, et al. Prevalence of allergic disorders in Italy: the Cotignola population study. Eur Ann Allergy Clin Immunol. 2012;44(1):5-11

4. Asher M, Montefort S, Björkstén B, et al; ISAAC Phase Three Study Group. Worldwide time trends in the prevalence of symptoms of asthma, allergic rhinoconjunctivitis, and eczema in childhood: ISAAC Phases One and Three repeat multicountry cross-sectional surveys. Lancet. 2006;368(9537):733-743.

5. Worldwide variation in prevalence of symptoms of asthma, allergic rhinoconjunctivitis, and atopic eczema: ISAAC. The International Study of Asthma and Allergies in Childhood (ISAAC) Steering Committee. Lancet. 1998;351(9111):1225-1232.
6. Galassi C, De Sario M, Biggeri A, et al. Changes in prevalence of asthma and allergies among children and adolescents in Italy: 1994-2002. Pediatrics. 2006;117:34-42.

7. Brescianini S, Brunetto B, Iacovacci P, et al. Prevalence of self-perceived allergic diseases and risk factors in Italian adolescents. Pediatr Allergy Immunol. 2009;20:578-584.

8. Tozzi AE, Armenio L, Bernardini R, et al. Pediatric allergy and immunology in Italy. Pediatr Allergy Immunol. 2011;22(3):267-276.

9. Muraro A, Clark A, Beyer K, et al. The management of the allergic child at school: EAACI/GA2LEN Task Force on the allergic child at school. Allergy. 2010; 65(6):681-689.

10. Pols DHJ, Wartna JB, van Alphen EI, et al. Interrelationships between atopic disorders in children: a meta-analysis based on ISAAC questionnaires. PLoS ONE. 2015;10(7):e0131869.

11. Gupta R, Sheikh A, Strachan DP, Anderson HR. Burden of allergic disease in the UK: secondary analyses of national databases. Clin Exp Allergy. 2004;34:520-526.

12. Galimberti M, Passalacqua $\mathrm{G}$, Incorvaia $\mathrm{C}$, et al. Catching allergy by a simple questionnaire. World Allergy Organ J. 2015;11;8(1):16.

13. Scientific Advisory Committee of the Medical Outcomes Trust. Assessing health status and quality-of-life instruments: attributes and review criteria. Qual Life Res. 2002;11:193-205.

14. Fayers P, Hays RD, Revicki DA. Reliability and validity, including responsiveness. In: Fayers P, Hays RD, editors. Assessing Quality of Life in Clinical Trials: Methods and Practice. 2nd ed. New York: Oxford University Press; 2005: 25-39.

15. Garcia de Yebenes Prous MA, Rodriguez Salvanes F, Carmona Ortells L. Validación de cuestionarios. [Validation of questionnaires]. Reumatol Clin. 2009;5:171-177. Spanish.

16. Castro-Rodriguez JA. The Asthma Predictive Index: a very useful tool for predicting asthma in young children. J Allergy Clin Immunol. 2010;126:212-216.

17. Pescatore AM, Dogaru CM, Duembgen L, et al. A simple asthma prediction tool for preschool children with wheeze or cough. J Allergy Clin Immunol. 2014;133(1):111-118.

18. Redline S, Larkin EK, Kercsmar C, Berger M, Siminoff LA. Development and validation of school-based asthma and allergy screening instruments for parents and students. Ann Allergy Asthma Immunol. 2003;90(5):516-528.

19. Redline S, Gruchalla RS, Wolf RL, et al. Development and validation of school-based asthma and allergy screening questionnaires in a 4-city study. School Nurse News. 2004;21(5):12-14.

20. Jones A, Bowen M. Screening for childhood asthma using an exercise test. Br J Gen Pract. 1994;44(380):127-131.

21. Bauer EJ, Lurie N, Yeh C, Grant EN. Screening for asthma in an innercity elementary school in Minneapolis, Minnesota. J Sch Health. 1999;69:12-16.

22. Savenije OEM, Kerkhof M, Koppelman GH, Postma DS. Predicting who will have asthma at school age among preschool children Review Article. J Allergy Clin Immunol. 2012;130:325-331.

23. Bonini M, Braido F, Baiardini I, et al. AQUA: Allergy Questionnaire for Athletes. Development and validation. Med Sci Sports Exerc. 2009;41(5): 1034-1041.

24. Sacchetti M, Baiardini I, Lambiase A, et al. Development and testing of quality of life in children with Vernal Keratoconjunctivitis questionnaire. Am J Ophthalmol. 2007;144:557-563.

25. Ducharme FM, Tse SM, Chauhan B. Diagnosis, management, and prognosis of preschool wheeze. Lancet. 2014;383:1593-1604.

26. Murphy KR, Zeiger RS, Kosinski M, et al. Test for respiratory and asthma control in kids (TRACK): a caregiver-completed questionnaire for preschool-aged children. JAllergy Clin Immunol. 2009;123:833-839.

27. Sato K, Sato Y, Nagao M, et al. Development and validation of asthma questionnaire for assessing and achieving best control in preschool-age children. Pediatr Allergy Immunol. 2016;27(3):307-312.

28. Savitz DA. Interpreting Epidemiologic Evidence: Strategy for Study Design and Analysis. Oxford, UK: Oxford University Press; 2003.

29. Soriano JB, Rabe KF, Vermeire PA. Predictors of poor asthma control in European adults. J Asthma. 2003;40(7):803-813. 
Pediatric Health, Medicine and Therapeutics is an international, peerreviewed, open access journal publishing original research, reports, editorials, reviews and commentaries. All aspects of health maintenance, preventative measures and disease treatment interventions are addressed within the journal. Practitioners from all disciplines are invited to submit their work as well as healthcare researchers and patient support groups. The manuscript management system is completely online and includes a very quick and fair peer-review system. Visit http://www.dovepress.com/ testimonials.php to read real quotes from published authors.

Submit your manuscript here: http://www.dovepress.com/pediatric-health-medicine-and-therapeutics-journal 